\title{
La guerre iroquoise et la mortalité en Nouvelle-France, 1608-1666
}

\section{John A. Dickinson}

Volume 36, numéro 1, juin 1982

URI : https://id.erudit.org/iderudit/304030ar

DOI : https://doi.org/10.7202/304030ar

Aller au sommaire du numéro

Éditeur(s)

Institut d'histoire de l'Amérique française

ISSN

0035-2357 (imprimé)

1492-1383 (numérique)

Découvrir la revue

Citer cet article

Dickinson, J. A. (1982). La guerre iroquoise et la mortalité en Nouvelle-France, 1608-1666. Revue d'histoire de l'Amérique française, 36(1), 31-54.

https://doi.org/10.7202/304030ar d'utilisation que vous pouvez consulter en ligne.

https://apropos.erudit.org/fr/usagers/politique-dutilisation/ 


\title{
LA GUERRE IROQUOISE ET LA MORTALITÉ EN NOUVELLE-FRANCE 1608-1666
}

\author{
JOHN A. DICKINSON \\ Département d'histoire \\ Université de Montréal
}

Malgré les progrès réalisés dans les dernières années pour arriver à une vision plus nuancée de la réalité amérindienne ${ }^{1}$, plusieurs manuels et oeuvres de vulgarisation continuent de véhiculer le vieux stéréotype du barbare assoiffé de sang, dont le seul rôle dans l'histoire est de massacrer les pauvres colons ${ }^{2}$. Ainsi, dans l'imagination populaire, l'âge hérö̈que de la Nouvelle-France est perçu comme une période de rudes épreuves pour les pionniers de la colonie. Assiégés continuellement par les Iroquois, ils vivaient sous la menace constante d'être assassinés dans leurs champs ou d'être emmenés captifs pour subir un sort plus cruel en Iroquoisie. Ces images conditionnent encore notre perception des Amérindiens. Cependant, on ne s'est guère attardé à la portée précise de la guerre iroquoise sur la mortalité à cette époque. Seule une analyse détaillée de tous les décès causés par les Iroquois permettra de déterminer si l'image traditionnelle est fondée sur les faits ou sur la généralisation hâtive d'exemples célèbres (le martyre de Jean de Brébeuf par exemple) maintes fois cités, mais peu représentatifs. Une telle analyse aidera aussi à mieux saisir le comportement des Iroquois et à déterminer les objectifs qu'ils poursuivaient.

L'évolution du «sauvage» dans l'historiographie a été retracée par Donald B. Smith, Le "Sauvage» pendant la période héroïque de la Nouvelle-France (1534-1663) d'après les historiens canadiens-français (Montréal, Hurtubise HMH, 1979).

2 Le ton des manuels est devenu plus neutre au point d'en faire presque disparấtre les autochtones sauf lorsqu'il s'agit des guerres iroquoises. Un exemple récent de ce traitement se trouve dans Michel Allard et al, Histoire nationale du Québec, de sa découverte à aujourd'hui (Montréal, Guérin, 1980). L'image de l'Amérindien que l'on trouve dans une vulgarisation très populaire (Nos racines. L'histoire vivante des Québécois, Les éditions T.L.M., 1979) est également négative. Le fascicule numéro 8 intitulé «les guerres iroquoises» perpétue l'image traditionnelle et insiste beaucoup trop sur la barbarie des guerriers. On peut y lire que les Iroquois sont «une menace constante» et que la torture est «une denrée quotidienne». En renforçant les vieux préjugés et en citant des exemples spectaculaires de cruauté on ne contribue guère à promouvoir une meilleure compréhension entre Québécois d'origine amérindienne et Québécois d'origine européenne. 
Les sources pour l'étude des victimes des Iroquois sont bien connues des historiens. Elles comprennent essentiellement les écrits des Jésuites (les Relations et le Journal), les registres d'état civil des paroisses de Québec, de Trois-Rivières et de Montréal, la correspondance de Mère Marie de l'Incarnation, l'Histoire du Canada du jésuite François Ducreux et les Oeuvres de Champlain. La valeur de ces sources est cependant assez inégale, et il faut préciser les apports comme les lacunes de chacune d'entre elles.

Les Relations des Jésuites constituent le tableau le plus complet des événements importants survenus dans la colonie au cours de chaque année. Rédigées l'automne, avant le départ des vaisseaux, par le supérieur de la mission canadienne puis publiées à Paris, elles servaient à faire connaître la colonie dans la métropole. La description des événements est souvent très détaillée; surtout lorsqu'il s'agit d'une mort édifiante. Cependant, les détails précis (le nom des personnes impliquées, le sort des captifs) sont parfois négligés. Il n'existe qu'une lacune importante: la Relation de 1655 perdue lorsque le messager à qui on l'avait confiée fut volée entre La Rochelle et Paris ${ }^{3}$. A partir de 1663, les indications sur les pertes françaises sont assez maigres, mais les autres sources indiquent que ces années n'étaient pas très importantes de ce point de vue. Ainsi peut-on connaître le nombre total des victimes la plupart du temps, mais il faut chercher ailleurs pour compléter les détails concernant les noms des victimes et les circonstances des attaques.

Le Journal des Jésuites fut rédigé au jour le jour par le supérieur à Québec. Il commence le 17 octobre 1645 et ne comporte qu'une lacune sérieuse: du 5 février 1654 au 25 octobre 1656, le Journal tenu dans un cahier in quarto est maintenant perdu ${ }^{4}$. Il a l'avantage de décrire les premières réactions devant les événements et constitue une excellente source sur tous les faits divers. Cependant, comme il fut rédigé à Québec il y a toujours le danger de sous-évaluer les événements qui se sont déroulés dans les autres régions de la colonie. Le risque existe surtout pour Montréal, le bourg le plus éloigné. Effectivement, pour la période 1646-1660, le Journal enregistre $83,3 \%$ des événements survenus dans l'île et indiqués dans le registre paroissial. Trois-Rivières étant plus proche, la couverture devrait en être meilleure, et il semble probable qu'au moins $90 \%$ des événements survenus dans la région trifluvienne y soient inclus. Les registres paroissiaux de la NouvelleFrance et leurs actes de sépulture devraient normalement consti-

\footnotetext{
Relations des Jésuites (Montréal, Éditions du Jour, 1972): 1655: 1 (Ci-après $R J$ ).

Charles-Henri Laverdière et Henri-Raymond Casgrain, éds., Journal des Jésuites (Montréal, Editions François Xavier, 1973): 199 (Ci-après JJ).
} 
tuer la première source sur les décès à condition qu'ils aient été bien tenus. Celui de Montréal est exemplaire car il mentionne non seulement toutes les personnes tuées par les Iroquois sur l'île, mais aussi les captifs morts en Iroquoisie. Il a été possible de dresser une liste complète pour cette localité. Les données pour Trois-Rivières sont malheureusement trop incomplètes pour permettre une telle opération. Les registres de Québec ne sont guère plus riches, mais comme la plupart des victimes dans cette région furent tuées assez loin de l'église (à Cap-Rouge, sur la côte de Beaupré et à l'île d'Orléans), il ne semble pas que leurs corps y furent transportés pour l'inhumation.

La correspondance de Marie de l'Incarnation répète le plus souvent des renseignements fournis ailleurs par les Jésuites qui étaient ses principaux informateurs. Cependant, elle donne à l'occasion une information supplémentaire, et ses lettres aident à combler le vide causé par la perte du cahier du Journal et le vol de la Relation de 1655. Elle complète aussi les autres sources en confirmant des détails qui autrement ne seraient pas clairs. L'Histoire $d u$ Canada de François Ducreux est utile principalement pour les renseignements concernant l'année 1655. Enfin, les Oeuvres de Champlain constituent la meilleure source pour la période 16081632.

Ces sources permettent de dresser des listes, aussi exhaustives que possible, de tous les Français tués ou emmenés captifs par les Iroquois entre 1608 et $1662^{5}$. Pour les années 1663-1666, les indications sont plus fragmentaires et ne méritent pas la même confiance. Il est vraisemblable que la seule victime de 1663 soit connue puisque la Relation de cette année indique que seul Montréal «a été teint du sang de Français» ${ }^{6}$. En 1664 , les Iroquois auraient tué «dans nos champs divers Français» ${ }^{7}$. Outre les quatre victimes de Montréal, seul un dénommé André de Québec est connu. Au début de mai 1665 , les Jésuites reçurent la nouvelle de «plusieurs massacres faits à Montréal» ${ }^{8}$. Presque toutes les mentions d'événements pendant ces années concernent Montréal; il n'y eut qu'un mort à Québec et deux personnes capturées dans d'autres régions de la colonie, l'une à l'île d'Orléans et l'autre à Trois-Rivières en $1664^{\circ}$. À partir de 1665 , les forts sur le Richelieu protégeaient les

\footnotetext{
Voir Annexe I: Français tués par les Iroquois, 1608-1666; Annexe II: Captifs Français 1608-1666.

$R J$, 1663: 12 .

$R J, 1664: 29$.

$J J: 331$.

$R J, 1664: 29$.
} 
habitations sur le Saint-Laurent en aval de Sorel. Ni le Journal, ni les lettres de Marie de l'Incarnation ne mentionnent de victimes dans la région de Québec et il est logique de conclure que ce silence reflète la réalité. Il y en eut peut-être dans la région des TroisRivières, mais le nombre ne devait pas être important. Comme tous les décès à Montréal sont connus, il est très possible que la liste soit complète pour cette période. Toutefois le nombre de captifs pris à cette époque est impossible à déterminer. Les pertes militaires de 1666 sont mal indiquées et n'ont pas été incluses dans la compilation. D'après les renseignements de la Relation de 1666, le total ne devait pas dépasser la dizaine ${ }^{10}$.

Pour la période 1608 à 1662 , l'énumération des 59 personnes tuées par les Iroquois dans la région de Montréal est complète à l'exception du nom d'un ouvrier tué au fort Richelieu en 1644. Tous les détails sont connus sur les huit personnes qui trouvèrent la mort pendant qu'elles étaient en mission chez les Agniers ou chez les Hurons. Dans la région de Trois-Rivières il nous manque les noms d'un peu moins du tiers des 36 victimes. C'est dans cette région qu'existe un risque de sous-évaluation, mais nous croyons avoir pu repérer la grande majorité des cas. 17 des 38 victimes de la région de Québec restent dans l'anonymat, mais ce total doit correspondre à la réalité. Le total des victimes s'élève donc à 141 pour la période 1608-1662. Entre 1663 et 1666, les 11 victimes de la région de Montréal sont connues ainsi qu'une victime de la région de Québec.

Cette liste ignore certains cas marginaux. Les trois Français, noyés sur le Saint-Laurent lors de la retraite de la mission de Sainte-Marie de Gannentaha au printemps de $1658^{11}$, sont exclus puisque leur mort n'est pas directement reliée à une action des Iroquois. Les accidents de ce genre sur le fleuve étaient assez fréquents; 29 noyades sont dénombrées dans le Journal des Jésuites entre 1646 et $1662^{12}$. Par contre, Mathurin Soulard et Blaise Juillet

$10 R J, 1666: 5-9$. Les noms de trois officiers sont connus: les sieurs d'Aiguesmortes, de Traversy et de Clusy. D’Aiguesmortes fut tué en compagnie de «quelques-uns de nos soldats». Les soldats faits prisonniers lors de l'attaque contre de Traversy et de Clusy furent ramenés immédiatement par des ambassadeurs «qui venoient offrir toute sorte de satisfaction pour le meurtre de ceux qui avoient esté tués, et de nouvelles suretés pour la paix». Le sieur de Luques et sept soldats se noyèrent dans le lac Champlain pendant la campagne de cette année. Pour un militaire en campagne la mort fait partie des risques professionnels; c'est pour cette raison que ces hommes ne sont pas inclus dans notre compilation.

$11 R J, 1658: 9$.

$12 \mathrm{JJ}$, passim. A Montréal, 9,8\% des décès sont causés par la noyade entre 1642 et 1663. Marcel Trudel, Montréal, la formation d'une société 1642-1663 (Montréal, Fides, 1976), 32. Il est à noter que nos chiffres ne correspondent pas nécessairement avec ceux de Trudel, puisque les captifs tués sont énumérés à part. 
ont été inclus puisque leur noyade en 1660 peut être attribuée à la panique causée par l'irruption d'une bande iroquoise. Le serviteur d'Antoine des Rosiers nommé Mathurin, tué en août 1651, de bonne heure le matin lorsqu'il chassait des corneilles près de TroisRivières, a été inclus sous toutes réserves. On présume que les Iroquois sont responsables de sa mort mais les preuves concrètes manquent. Michel Louvard qui fut tué le 23 juin 1662 «sur le pas de sa porte on ne sait par qui si ce n'est les sauvages Loups» ${ }^{13}$ n'a pas été inclus. Les deux enfants Poulain qui disparurent de la côte de Beaupré en juin $1661^{14}$ se sont vraisemblablement perdus dans les bois. Il est possible qu'ils aient été capturés, mais il ne faudrait pas prendre pour acquis que les Iroquois sont automatiquement responsables de chaque disparition.

L'étude des captifs pose plus de problèmes. Le nombre est assez souvent indiqué avec précision, surtout pour les années antérieures à 1663 . En effet, on considérait le plus souvent les captifs comme des disparus voués à une mort certaine aux mains des Iroquois. Pour cette raison, nous croyons que les 133 personnes amenées en Iroquoisie pendant ces années constituent la presque totalité des gens impliqués. À partir de 1663, la recension est moins précise et les dix personnes énumérées ne sont qu'une partie du total. Cependant, la majorité des captifs de cette époque sont libérés pour faire avancer les pourparlers de paix. Aussi, comme Montréal est le principal théâtre des activités iroquoises et que les registres paroissiaux indiquent les décès en Iroquoisie, il est vraisemblable que toutes les victimes de cette période sont connues. Malheureusement, seulement un peu plus de $40 \%$ des captifs ont pu être identifiés d'une manière certaine. Quant à leur sort, il a pu être déterminé dans $79 \%$ des cas. Parmi ceux-ci, 14 sont certainement tués et 24 autres le sont probablement. La guerre iroquoise est donc directement responsable de la mort de 191 Français entre 1608 et 1666 .

Comme l'a déjà remarqué Marcel Trudel à propos de Montréal la mortalité causée par les Iroquois ne frappe pas d'une façon continue ${ }^{15}$; ses variations traduisent l'évolution des rapports entre Français et Iroquois. La guerre entre nations amérindiennes sur le territoire de la Nouvelle-France est continuelle pendant toute la période, mais les Français n'y sont impliqués qu'épisodiquement. Traditionnellement, la guerre iroquoise débute en 1641 et se

13 Registres de Notre-Dame de Montréal. Trudel note neuf décès causés par les Iroquois pendant cette année. Trudel, Montréal, 32. Nous n’avons pu trouver que six morts à Montréal et un en captivité. M. Trudel a-t-il compté Louvard?

14 JJ: 297.

15 Trudel, Montréal, 30. 
poursuit sans relâche pendant 26 ans ${ }^{16}$. Trudel vient de proposer une nouvelle chronologie: une première guerre entre 1647 et 1653 et une seconde qui débute en $1657^{17}$. Toutefois, même cette chronologie traduit mal les attaques dirigées contre des Français. L'analyse de la répartition chronologique des événements suggère six phases distinctes (Voir Tableau I).

\begin{tabular}{lccccccc}
\hline \multicolumn{8}{c}{ TABLEAU I } \\
& Répartition chronologique des événements & \\
Période & $1608-41$ & $1642-49$ & $1650-53$ & $1654-59$ & $1660-61$ & $1662-66$ & Total \\
Tués & 4 & 18 & 32 & 19 & 58 & 22 & 153 \\
Captifs & 2 & 14 & 25 & 32 & 59 & 11 & 143 \\
\hline Total & 6 & 32 & 57 & 51 & 117 & 33 & 296 \\
& & & & & & & \\
\hline
\end{tabular}

Dans un premier temps, les Français n'intéressent pas beaucoup les Iroquois. Entre 1608 et 1641, seulement quatre victimes et deux captifs sont dénombrés. La construction du fort Richelieu et l'établissement de Montréal, en 1642, ouvrent une ère nouvelle dans les relations franco-iroquoises en posant une menace pour les lignes de communication des Agniers. Des affrontements sporadiques marquent les années 1642-1649. Cependant les Iroquois sont encore préoccupés par les Hurons à cette époque. La première vraie guerre éclate en 1650 et dure jusqu'en 1653. Une trêve assez incertaine suit entre 1654 et 1659 . Le combat du Long-Sault inaugure une nouvelle période d'hostilités intenses mais de courte durée. À partir de 1662, les affrontements diminuent en intensité; les Iroquois sont déterminés à maintenir une certaine pression sur la colonie tout en négociant une paix durable.

S'il est vrai que la mortalité ne frappe pas d'une façon continue, il est également vrai que la distribution géographique des attaques iroquoises évolue de façon significative (voir la carte). Montréal a souvent tenu la vedette dans l'historiographie traditionnelle, grâce surtout à l'influence de l'Histoire du Montréal du sulpicien François Dollier de Casson qui constitue l'une des «sources» privilégiées des abbés Faillon et Groulx. Certes, Montréal dut pleurer plus de morts que les autres centres, mais le nombre de captifs y est de loin inférieur à celui de la région de Trois-Rivières. L'effectif des gens touchés par la guerre est presque égal dans les deux régions:

16 Émile Salone. La colonisation de la Nouvelle-France (Trois-Rivières, Boréal Express, 1970), 85-86.

17 Marcel Trudel, Histoire de la Nouvelle-France, Volume III, La seigneurie des Cent-Assciés, tome 1, Les événements (Montréal, Fides, 1979): 196-204; 225-269. 
DISTRIBUTION GÉOGRAPHIQUE DES FRANÇAIS TUÉS ET CAPTURÉS, 1608-1666

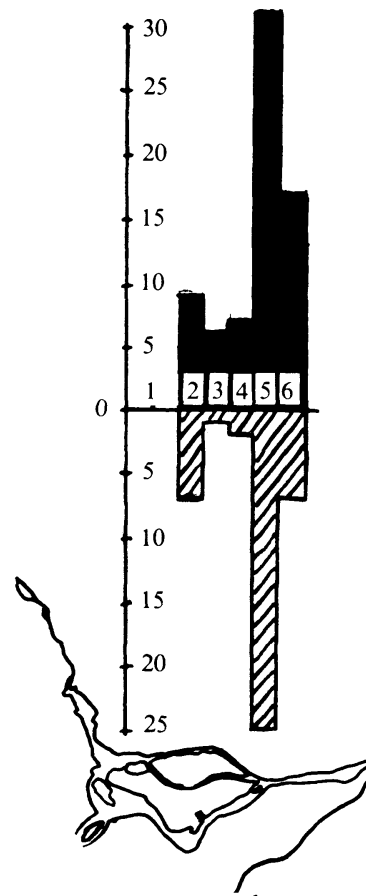

MONTRÉAL

1- 1608-1641

2- $1642-1649$

3- $1650-1653$
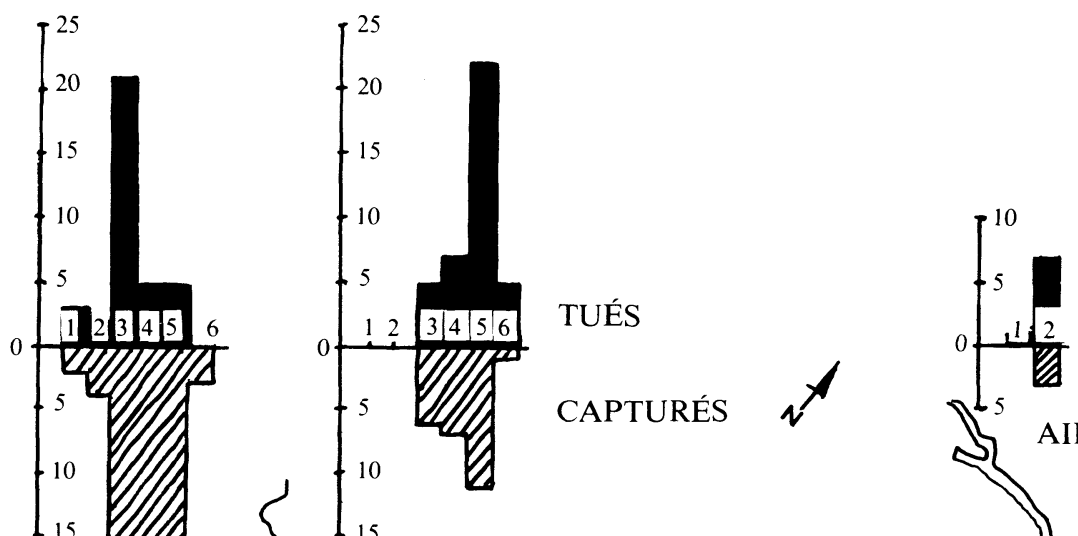

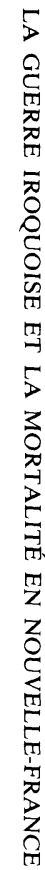

4- 1654-1659

5- 1660-1661

6- 1662-1666 
112 pour Montréal et 108 pour Trois-Rivières (Thomas Godefroy fut capturé deux fois). Les environs de Québec jouissaient d'une plus grande sécurité et ne comptent que 64 personnes impliquées. Enfin cinq Français furent tués lorsqu'ils étaient en mission chez les Hurons, trois autres périrent en mission chez les Agniers et trois furent capturés sur l'Outaouais, dont Isaac Jogues qui figure aussi parmi les trois victimes en mission chez les Agniers.

Malgré sa position d'avant-poste, Montréal se tire assez bien d'affaire jusqu'à la période 1660-1661. En effet, la moitié des pertes subies par cette localité se concentre dans ces deux années. À partir de 1662, Montréal demeure le seul centre vraiment menacé: un fait qui présage déjà la deuxième guerre iroquoise de 1689 à 1701. Un autre phénomène mérite d'être signalé: le déséquilibre entre le nombre de morts et de prisonniers. Malgré leur plus grande proximité de l'Iroquoisie, les Montréalais se font tuer plutôt qu'amener captifs. Ceci semble être dû à une plus grande discipline; ils s'aventurent rarement seuls hors de l'enceinte et sont armés pour pouvoir résister à une embuscade ${ }^{18}$. Dans ces conditions, les Iroquois ont du mal à faire des prisonniers. Près de la moitié des captifs sont pris lors du raid du début du printemps 1661. Devant le nombre et la détermination des Iroquois à cette occasion, rien ne pouvait prévenir le coup.

À Trois-Rivières, la pression est plus constante. La présence de nombreux Algonkins et le fait que ce bourg soit le point névralgique de la traite attiraient les Iroquois, et les risques étaient grands pour les Français qui chassaient dans les bois aux alentours, voire même pour ceux qui pêchaient dans le fleuve, devant le fort. Cependant, à part les années de «siège» (1652-1653), les victimes tuées sur-le-champ étaient rares. Les Trifluviens étaient les captifs par excellence et servaient de monnaie d'échange contre les Agniers et Onneyouts mis aux fers par les Français.

La région de Québec fut complètement épargnée jusqu'en 1650. Les premières attaques coïncident avec le début de la première véritable offensive contre les Français. Durant la trêve, de 1654 à 1659 , la présence sur l'île d'Orléans de ce qui reste de la nation huronne entraîne un harcèlement constant et explique les pertes comparativement élevées. Comme à Montréal, l'année 1661 fut désastreuse et compte $43,5 \%$ de tous les gens impliqués. Le peu de prisonniers s'explique par la distance entre Québec et les villages iroquois et les problèmes de transport qu'elle entraîne. Autre fait à

18 Il est probable que l'ordonnance de Chomedey de Maisonneuve de 1658 ne fait que confirmer officiellement un ordre verbal. Étienne-Michel Faillon, Histoire de la colonie française au Canada (Villemarie, Bibliothèque paroissiale, 1866), II: 384-385. 
signaler, le bourg de Québec sort pratiquement indemne; sept individus seulement sont tués dans la proche banlieue. La majorité des victimes de cette région se trouve sur l'île d'Orléans, sur la côte de Beaupré, à Cap-Rouge et à Tadoussac. La concentration de la population française à Québec et la présence de navires dans la rade pendant une partie de l'été expliquent sans doute la sécurité élevée.

Lorsque l'on pense aux victimes des Iroquois, l'image qui vient spontanément à l'esprit est celle du pionnier seul qui se fait surprendre dans ses champs par un ennemi caché dans le creux d'un arbre depuis quelques jours ${ }^{19}$. Cela arrive, mais ce genre d'attaque est loin d'être le modus operandi le plus commun. Les circonstances entourant la mort de 115 des 141 victimes entre 1627 et 1662 ont pu être déterminées. Seulement cinq personnes étaient seules et elles étaient parties à la chasse dans trois cas. Pendant cette période, les Français devaient être conscients du risque qu'il y avait à se promener seul. Les guerriers iroquois opéraient en bandes de 20 hommes ou plus et préféraient s'attaquer à des groupes pour pouvoir capturer des prisonniers. Des petits groupes de trois à cinq personnes qui travaillaient aux champs ou faisaient une charpente étaient de bonnes cibles. Le cas de Nicolas Godet, Jean Saint-Père et Jacques Noël, tués à Montréal en octobre $1657^{20}$, est assez typique de ce genre d'opération qui provoque au total 23 victimes. Même les groupes plus importants n'étaient pas à l'abri. L'attaque contre une équipe de 14 hommes partis chercher des matériaux sur l'île à la Pierre, en face de Montréal, en 1661, est exemplaire. Surpris sur l'île, le sulpicien Guillaume Vignal et trois ouvriers furent tués; Claude de Brigeart, René Cuillerier et un dénommé Dufresne furent faits prisonniers. Les autres réussirent à regagner Montréal ${ }^{21}$. Au total 26 victimes sont tuées lorsqu'elles sont dans des groupes de six personnes ou plus.

42 personnes sont tuées lors de véritables batailles. Dans la grande majorité des cas les Français manifestent une certaine négligence. Des sorties téméraires contre des forces ennemies supérieures en nombre sont la cause de trois décès en $1644^{22}$, de sept en 1661, dont le sénéchal Jean de Lauson ${ }^{23}$, et de la mort de Lambert

\footnotetext{
19 Le «tableau» dépeint par Henri-Raymond Casgrain dans «Les pionniers canadiens» traduit à merveille cette image. Henri-Raymond Casgrain, Oeuvres complètes (Montréal, Beauchemin, 1866), tome I: 54-55.

20 François Dollier de Casson, Histoire du Montréal (Ralph Flenley éd.), (London, J.M. Dent \& Sons, 1928), 220. re, 280-284.

21 RJ, 1665: 20; Registres de Notre-Dame de Montréal; Dollier de Casson, Histoi-

22 C'est la fameuse sortie de Chomedey de Maisonneuve avec 29 compagnons contre au moins 80 Iroquois. $R J, 1644$ : 42; Dollier de Casson, Histoire, 118-120.

23 RJ, 1661: 4-5; JJ: 298-299.
} 
Closse et trois de ses compagnons en $1662^{24}$. Les erreurs tactiques sont encore plus meurtrières. Le gouverneur de Trois-Rivières, Guillaume Guillemot du Plessis-Kerbodot, mit le pied à terre dans «un lieu plein de vases et fort désavantageux» lorsqu'il poursuivait des Iroquois en 1652. Cette bévue coûta la vie à huit Français et entraîna la capture de sept autres ${ }^{25}$. En 1660, Dollard et ses compagnons, plutôt que de rechercher un meilleur site défensif, se sont enfermés dans un petit fort «qui n'était pas de défense» et où il manquait de l'eau potable. De plus, ils tirèrent sur les Iroquois lorsque les pourparlers étaient encore en cours ${ }^{26}$.

15 personnes périrent en compagnie d'Amérindiens. Les missionnaires jésuites constituent la majorité de celles-ci. Trois personnes furent assassinées lorsqu'elles étaient en ambassade en Iroquoisie. Il s'agit de Pierre Magnan, tué avec des ambassadeurs algonkins par les Agniers en $1627^{27}$ et du père Isaac Jogues accompagné de Jean La Lande, torturés lors d'une mission en $1646^{28}$. Enfin, Noël Godin fut surpris lorsqu'il pêchait dans le fleuve devant Trois-Rivières en 1651. Son compagnon, Lajeunesse, réussit à regagner le fort avec un bras cassé et une épaule transpercée d'une balle ${ }^{29}$. La vaste majorité des Français meurent lorsqu'ils sont en groupe, lorsqu'ils participent à un affrontement militaire ou lorsqu'ils accompagnent les autochtones qui sont continuellement en guerre contre les Iroquois.

Le sort réservé aux captifs français subit une évolution assez marquée à travers toute la période (voir Tableau II).

\section{TABLEAU II \\ Destin des captifs français}

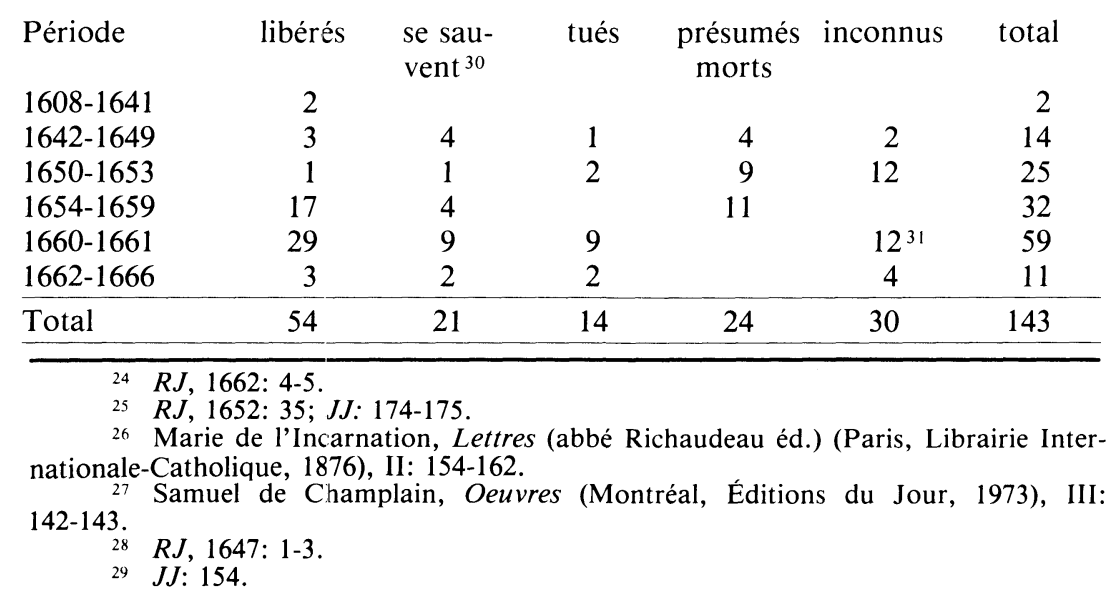


Les deux premiers Français à connaître la captivité furent pris par hasard en 1641. Les Iroquois, suivant des traces de raquettes, tombèrent sur Thomas Godefroy et François Marguerie qu'ils pensaient être des Algonkins. Dès qu'on s'aperçut qu'ils étaient des Blancs «ils ne les traitèrent pas comme ils font les sauvages, usans d'une plus grande douceur; car ils ne leur arracherent ny les ongles des doights, ny ne les meurtrirent en aucune partie de leur corps $)^{32}$. À cette époque, les Iroquois n'avaient pas encore décidé de s'engagner dans des hostilités avec les Français et ils n'avaient pas encore de morts à venger ${ }^{33}$. La décision des Français d'aider activement leurs alliés amérindiens se manifesta par la construction d'un fort à l'embouchure du Richelieu. Pour protéger leurs lignes de communication, les Iroquois attaquèrent les ouvriers dépêchés sur les lieux. Ces actions allaient transformer les enjeux.

Pendant la période 1642-1649, 14 Français furent emmenés dans des villages iroquois. Le sort qui attendait ceux qui ne réussirent pas à s'évader était plus cruel que celui réservé à Godefroy et à Marguerie. Même ceux qui étaient libérés par la suite subissaient les «tourments» de leurs «hôtes» avant de partir ${ }^{34}$. Avec le déclenchement des hostilités ouvertes en 1650, la captivité signifiait une mort presque certaine. Les Iroquois avaient de plus en plus de morts à venger, et il fallait apaiser les esprits des guerriers disparus. Plusieurs Français furent tués, mais il ne faut jamais oublier que les Iroquois subissaient aussi des pertes dans les engagements. Catherine Mercier de Montréal fut brûlée après un supplice horrible pour «venger la mort de huit de leurs hommes qui estoient demeurez dans un combat cet esté» ${ }^{35}$. Étant donné le destin réservé à ceux dont le sort est connu, il semble raisonnable de conclure que les 12 inconnus furent tous tués à l'exception du petit Charles Garman dit LePicard, qui est sans doute le petit garçon réclamé par le gouverneur en $1659^{36}$.

30 Deux captifs qui se sauvent se perdent dans les bois et ne rentrent jamais en Nouvelle-France. Cependant il est possible qu'ils aient réussi à gagner la Nouvelle-Hollande ou la Nouvelle-Angleterre.

31 Pour 1661, nous connaissons l'identité des deux Montréalais qui ont été tués. Tous les autres ont dû être libérés. Ainsi parmi les 29 personnes libérées figurent nécessairement les Montréalais pris en août et en octobre. Il est possible que Liberté, Antoine de la Meslé, Hébert et Pierre Rencontre faisaient partie des captifs du printemps. Dans ce cas, le destin de six captifs seulement serait inconnu et le total serait réduit à 54 pour 1660-1661 et à 139 pour l'ensemble.

32 RJ, 1641: 38. avaient été oubliés.

Ceci indique que les Iroquois tués par Champlain une génération auparavant

${ }_{34}$ Ce fut le cas pour Guillaume Couture et pour le père Bressani. $R J, 1643: 76 ; R J$, 1644: 41-45. On peut présumer que le traitement accordé à Pierre Lefebvre ne fut guère mieux.

\footnotetext{
$35 R J, 1651: 2$.

36 JJ: 256.
} 
La conception iroquoise de la paix inaugurée en novembre 1653 ne fut jamais comprise par les autorités françaises et, notamment, par les Jésuites qui soupçonnaient leurs anciens ennemis d'utiliser cet armistice pour cacher des desseins belliqueux. Cependant, à en juger par leur comportement envers les captifs, du moins jusqu'en 1659, les Iroquois étaient sincères dans leur intention de maintenir des rapports paisibles avec les Français. Tous les captifs de la période 1654-1658 furent libérés ou réussirent à s'échapper, à l'exception d'un dénommé Fouquet pris à TroisRivières en 1658. Les Iroquois demeuraient hostiles envers les alliés amérindiens et surtout envers les Hurons, mais ils ne cachaient pas cette animosité. Ceci avait pour conséquence des incidents fâcheux, à cause de la proximité des habitations françaises, mais, à chaque occasion, des délégations arrivaient pour tenter une réconciliation à la manière amérindienne: des présents étaient offerts en réparation pour le sang répandu ${ }^{37}$. Pour les Français, cependant, il s'agissait de meurtres, et la réparation n'était pas considérée comme une manière acceptable de régler un tel crime. Les mésententes provenaient de deux conceptions différentes de la justice. Le 21 septembre 1657, d'Ailleboust prit la décision de riposter, et les Iroquois trouvés près des habitations françaises furent capturés et mis aux fers ${ }^{38}$. D'Argenson continua dans la même voie après son arrivée en 1658, et de plus en plus d'Agniers et d'Onneyouts furent pris par «finesse». De plus, trois Agniers furent tués en septembre $1658^{39}$, et 11 autres en $1659^{40}$. Devant l'escalade des actions hostiles les Iroquois réagirent en 1659 . Des 12 personnes capturées cette année-là, deux seulement réussirent à s'évader. Les autres furent vraisemblablement torturées pour venger la mort et la capture des Agniers dans la vallée laurentienne ${ }^{41}$. Ce revirement dans le traitement des prisonniers est très net et il présage les événements de 1660-1661, les pires années pour la colonie.

Une armée d'environ 500 Agniers et Onneyouts partit au printemps de 1660 pour le Saint-Laurent. Vraisemblablement, elle voulait en finir avec les Hurons et peut-être capturer des Français pour servir d'otages dans les discussions de paix. Quoi qu'il en soit, elle ne put poursuivre son but puisqu'elle dut rebrousser chemin pour aider des chasseurs onontagués et tsonnontouans aux prises avec Annaotaha et Dollard au Long-Sault ${ }^{42}$. Les pertes iro-

37 Ce fut le cas pour le père Gareau tué en 1656 et encore pour les trois Montréalais tués en 1657. Marie de l'Incarnation, Lettres, II: 86-88; $R J, 1658$ : 10.

38 JJ: 221-225; $R J, 1658: 10-11$

39 JJ: 243.

40 Marie de l'Incarnation, Lettres, II: 140.

4 JJ: 259-263; RJ, 1659: 2.

42 John A. Dickinson, "Annaotaha et Dollard vus de l'autre côté de la palissade», Revue d'histoire de l'Amérique française, 35,2 (septembre 1981): 163-178. 
quoises auraient été de 14 hommes tués et de 19 blessés ${ }^{43}$. Ces pertes devaient être vengées, et les captifs pris par la suite couraient un grand risque. Cependant, le pire ne se produisit pas. Des 59 prisonniers capturés au cours des années 1660 et 1661, au moins 38 eurent la vie sauve. Parmi ceux qui furent tués, figurent Marie Caron blessée à mort lorsque les Algonkins tentèrent de la sauver ${ }^{44}$ et le dénommé Liberté assassiné parce qu'il refusa de prendre une femme iroquoise ${ }^{45}$. La majorité des prisonniers furent «traités comme leurs enfants» ${ }^{46}$. En effet, une activité diplomatique intense débuta en 1661 et, pour prouver leur bonne foi, les ambassadeurs ramenèrent des captifs. Le chiffre de plus de 70 victimes donné par les Jésuites pour 1661 serait donc considérablement au-delà de la réalité, puisque ce nombre inclut les captifs encore détenus dans les villages iroquois au moment où la Relation est rédigée ${ }^{47}$.

Les données sur les captifs de la période 1662-1666 sont éparses. Cependant, l'activité diplomatique se poursuit pendant ces années et il est vraisemblable qu'à part les deux Montréalais brûlés en Iroquoisie, les autres furent libérés. Nous pouvons répartir ceux dont le destin est inconnu comme suit: 1642-1649, deux présumés morts; 1650-1653, 11 présumés morts; 1660-1661, 12 présumés libérés; 1662-1666, quatre présumés libérés. Ces hypothèses donneraient un grand total de 51 captifs morts en Iroquoisie. Plus de précision aurait été souhaitable, mais ces résultats donnent des ordres de grandeur qui sont certainement proches de la réalité.

La mortalité due aux guerres iroquoises entre 1608 et 1666 est plus faible que ce que l'historiographie traditionnelle nous faisait appréhender: 191 disparitions certaines, et probablement 204 en comptant les captifs dont le destin est inconnu. Même en ajoutant les pertes militaires, on aurait du mal à atteindre 225 personnes. De plus, cette mortalité ne frappe pas de façon constante. À part deux courtes périodes (1650-1653 et 1660-1661), les assassinats commis par les Iroquois ne sont qu'une cause de décès parmi d'autres. Certaines années, le nombre de noyades et d'autres décès accidentels dépasse largement les pertes attribuables aux Iroquois. Pour situer

43 Jacques Rousseau, "'L'affaire Dollard' de Fort Orange au Long-Sault», Revue d'histoire de l'Amérique française, 14, 3 (décembre 1960): 374-375.

$44 \quad R J, 1660: 32-33$

$45 \quad R J, 1662: 14$

46 Marie de l'Incarnation, Lettres, II: 213; RJ, 1662: 13-17.

$47 R J, 1661: 6$. Au départ des vaisseaux, il y avait eu 38 décès et il restait encore 37 captifs sur les 51 de l'année (un s'était sauvé et 13 autres avaient été déjà libérés). Les Jésuites avaient l'habitude de présumer que les captifs étaient voués à une mort certaine jusqu'à preuve du contraire. Leur compte est donc justifié puisque 75 personnes étaient mortes ou en danger de périr. 
ces quelque 200 personnes dans une perspective plus vaste, il faudrait les comparer aux milliers d'Amérindiens qui périrent dans ces mêmes guerres ou qui succombèrent à des maladies importées par les Européens. Le compte n'a jamais été fait avec précision, mais il est certain que le nombre dépasse 50000 (si ce n'est pas 100 000) personnes. On n'a qu'à penser aux Hurons, Pétuns, Neutres, Eriés et aux Iroquoiens du Saint-Laurent qui furent anéantis.

D'un point de vue démographique, les pertes qui devraient normalement être les plus difficiles à supporter par la colonie sont sans doute celles des femmes. Avec deux fois plus d'hommes nubiles que de femmes en $1663^{48}$, la mort d'une épouse avait des dimensions bien plus tragiques que la disparition d'un mari ou d'un célibataire mâle. Les veuves pouvaient facilement trouver un nouveau conjoint, mais les chances des veufs étaient bien moindres. Cependant les femmes ne sont guère touchées par les incursions iroquoises. Seulement quatre cas sont connus: Catherine Mercier et Louise Cheneau tuées en 1651, madame Moyen en 1655 et Marie Caron en 1660. Il est possible que d'autres femmes figurent parmi les victimes de 1661 , mais le nombre est certainement inférieur à dix. Ainsi, la natalité est très peu affectée par la guerre.

La population de la colonie connaît une évolution vers la hausse pendant toute la période. Ainsi les pertes des premières années, alors que la population totale de la colonie est petite, sont plus durement ressenties que celles de la fin du régime des CentAssociés. Même si la moitié des gens sont tués après 1660, la colonie est beaucoup plus en mesure de passer à travers cette saignée puisque les morts ne représentent qu'environ $4 \%$ des effectifs. Au point de vue démographique, la survie de la colonie n'a été menacée que pendant les années 1650-1653. Les 32 morts et les 22 captifs que l'on présume morts étaient difficiles à supporter pour une population encore mal enracinée dans le territoire. En 1660, les acquis sont tels que même la perte d'environ 80 personnes ne pouvait remettre en question l'existence d'un peuplement français. Au point de vue économique, la menace était sérieuse; mais au point de vue démographique, il était inconcevable de tout abandonner.

Les incursions iroquoises auraient pu avoir d'autres effets néfastes sur le progrès du peuplement. En effet, des études récentes ont mis en lumière un phénomène important de cette période: le retour en France des engagés lorsque leur contrat était échu ${ }^{49}$. Il ne

48 Marcel Trudel, La population du Canada en 1663 (Montréal, Fides, 1973), 63.

49 Lucien Campeau estime que les deux tiers des hommes seuls rentraient en France. Lucien Campeau, Les Cent-Associés et le peuplement de la Nouvelle-France (Montréal, Bellarmin, 1974), 152. 
semble pas que la guerre ait été pour quelque chose dans cette décision, sauf pendant les années de 1650-1653, lorsqu'il y avait une véritable offensive dirigée contre la Nouvelle-France. C'est durant ces années difficiles que l'on retrouve la plupart des désertions. Qui plus est, le point le plus touché par les attaques, TroisRivières, est le centre le plus affecté par ce phénomène. En 1650, quatre serviteurs de $\mathrm{M}$. du Herisson prennent la fuite ${ }^{50}$. Au printemps de 1653, 16 hommes quittent Trois-Rivières et disparaissent avant que les Iroquois ne reviennent ${ }^{51}$. À la fin d'avril de la même année, deux serviteurs de Ruette d'Auteuil prennent la clé des champs ${ }^{52}$. Ils sont suivis, deux semaines plus tard, par Jacques Junier "qui s'éclipsa de Sillery» ${ }^{53}$. Le cas le plus curieux est celui d'un Français de Montréal qui se joint aux Iroquois en 1656 et qui serait, d'après Nicolas Perrot, responsable de la mort du père Gareau ${ }^{54}$. La Relation de 1656 confirme qu'un Français se trouvait parmi la bande iroquoise et qu'il fut livré aux autorités, pour ensuite être condamné au dernier supplice à Québec ${ }^{55}$. Enfin, en 1663 , neuf hivernants se sauvent de Tadoussac ${ }^{56}$. Dans ce cas, il est difficile de déterminer si c'est la crainte des Iroquois ou le désir de garder tout le profit de leur traite qui les a motivés. Globalement, les désertions étaient peu nombreuses et leur concentration dans le temps semble être significative. Cependant, comme la plupart des engagés travaillaient dans la région de Québec, la guerre ne les inquiétait pas outre mesure.

Plus que la menace iroquoise, ce sont des facteurs économiques qui déterminent l'évolution du peuplement. Avant 1645, le monopole de la traite n'encourageait pas l'établissement de colons; c'est pourquoi il n'y avait qu'environ 600 personnes dans la colonie à cette date ${ }^{57}$. L'avènement du régime de la Communauté des Habitants rendit la situation plus favorable, mais ce fut surtout la destruction de la Huronie en 1650 qui permit un essor marqué. Les Hurons étaient les principaux fournisseurs de denrées agricoles des tribus nomades qui vivaient de la chasse. Leur disparition créa un marché pour les surplus agricoles des habitants. Selon Simon Denys, les colons faisaient déjà de bonnes affaires dès 1651 , en échangeant du porc et surtout du blé d'Inde, du pain,

$50 \quad J J: 140$.

$J J: 178-179$

$J J: 179$.

$J J: 180$

54 Nicolas Perrot, Mémoires sur les moeurs, coutumes et relligion des sauvages de

l'Amérique septentrionale (J Tailhan éd.) (East Ardsley, S \& R Publishers, 1968), 84.

55 $R J, 1656: 44$

56 JJ: 318.

57 Trudel, Histoire, 159. 
des pois et des citrouilles contre des lots de castor ${ }^{58}$. Cet essor est aussi illustré par l'évolution de la distribution des terres en censive. Avant 1645, les résultats sont pitoyables. De 1645 à 1650, il y a une progression qui démontre que de plus en plus d'engagés se fixaient au pays. Cependant, c'est la période 1651-1655 qui fut la plus fructueuse: plus de 50000 arpents furent concédés et ceux-ci représentaient $52,8 \%$ de toutes les concessions effectuées sous le régime des Cent-Associés ${ }^{59}$. Enfin, la production agricole devenait rentable, et seul ce développement pouvait encourager les gens à s'établir définitivement au pays. Ainsi il est donc possible que les Iroquois aient contribué au développement démographique de la colonie. Quoi qu'il en soit, les conséquences de cette première guerre iroquoise sur le peuplement de la Nouvelle-France sont loin d'être dramatiques.

L'analyse des victimes permet aussi de fournir une explication du comportement iroquois. Le sort réservé aux captifs est très révélateur à cet égard. Jusqu'en 1642 , la guerre demeure un conflit traditionnel entre nations amérindiennes, et les Français n'y sont pas directement impliqués. Avec la décision de Montmagny d'appuyer ses alliés d'une manière plus concrète, les Français deviennent des cibles puisque les Iroquois se doivent de venger leurs guerriers tués par des Blancs. L'offensive sérieuse ne débute qu'en 1650. Cependant, il est peu probable que les Iroquois voulaient chasser les Français du pays; ils pouvaient très bien apprécier les avantages d'un deuxième comptoir pour concurrencer Fort Orange. Leur objectif semble plutôt de détruire complètement tous les alliés amérindiens pour forcer les Français à traiter avec eux. C'est cette explication qui donne un sens à la paix conclue en 1653 avec les Français, paix qui n'incluait ni les Algonkins ni les Hurons. Les meurtres commis entre 1654 et 1659 par une jeunesse indisciplinée étaient suivis par des délégations apportant des présents en réparation pour rétablir les bonnes relations selon la manière autochto$\mathrm{ne}^{60}$. Ce n'est qu'après la capture de plusieurs Iroquois par «finesse» que les choses se détériorèrent. En 1660 et 1661, les attaques reprenaient, mais encore une fois l'objectif n'était pas de chasser les Français de la colonie. Les bandes cherchaient à prendre un nombre considérable de prisonniers afin de forcer les autorités à conclure une paix plus ferme. Le comportement à l'égard des captifs de 1661 est révélateur à cet égard. Il est vraisemblable

58 Lucien Campeau, «Un témoignage de 1651 sur la Nouvelle-France», Revue d'histoire de l'Amérique française, 23,4 (mars 1970): 607.

59 Marcel Trudel, Les débuts du régime seigneurial au Canada (Montréal, Fides, 1974), 134-135.

60 $R J, 1658: 10$. 
que ceux qui meurent pendant ces opérations ainsi que ceux qui subissent la torture en Iroquoisie paient pour une résistance trop vigoureuse lors de leur capture. Comme toujours, les guerriers tombés pendant une campagne devaient être vengés. Après le raid massif de 1661, les Iroquois ne se montrent plus aussi hardis. D'une part, leurs forces, évaluées à 840 hommes en $1665^{61}$, sont inférieures au nombre d'hommes armés en Nouvelle-France. D'autre part, la famine, les maladies et les pertes subies dans leurs guerres les avaient affaiblis et démoralisés au point qu'ils ne pouvaient plus continuer les hostilités ${ }^{62}$.

L'image traditionnelle de cette guerre ne résiste pas à une analyse des faits. La cruauté toute relative des Iroquois était bien réelle, mais elle était dirigée le plus souvent contre d'autres nations amérindiennes. Même les Français les plus en péril échappaient au bûcher dans la majorité des cas. La menace pour les habitants n'était ni aussi constante ni aussi terrible qu'on a voulu le faire croire. Cette image s'applique peut-être davantage au deuxième conflit qui eut lieu entre 1689 et 1701 , mais les circonstances étaient bien différentes. Les Iroquois sont alors activement appuyés par l'impérialisme britannique qui cherche vraiment à expulser les Français du continent. Cependant, même en parlant de ce conflit, il faut se méfier des généralisations car toutes les actions se déroulent dans la région de Montréal. Ainsi plus de la moitié de la population échappe complètement aux horreurs de la guerre. Même dans ce gouvernement les effets sont très inégaux. Certaines paroisses et seigneuries subissent des pertes considérables tandis que d'autres sont épargnées.

${ }^{61} R J, 1665: 10-11$. Les Goyogouins (300 hommes) et les Tsonnontouans ( 1200 hommes) ne sont pas inclus puisque «ces deux dernières nations ne nous ont jamais fait la guerre ouvertement, et se sont toujours conservés comme neutres».

$62 R J, 1664: 33$. 
ANNEXE I

Français tués par les Iroquois

A. Région de Québec

\begin{tabular}{|c|c|c|c|c|}
\hline Date & Lieu de l'incident & Nombre & Noms connus & Source ${ }^{1}$ \\
\hline $11-05-50$ & Québec & 2 & & $J J: 137$. \\
\hline $13-05-50$ & Beauport & 1 & & $J J: 138$. \\
\hline $18-09-51$ & Sillery & 1 & Louise Cheneau & $J J: 160$. \\
\hline $10-06-53$ & Cap Rouge & 1 & François Boule & $J J: 181$ \\
\hline $10-11-54$ & Québec & 2 & $\begin{array}{l}\text { Guillaume Boesse } 2 \\
\text { Michel Morin }\end{array}$ & Registre de Québec \\
\hline $28-11-54$ & Québec & 1 & Pierre de Launé & Registre de Québec \\
\hline $27-05-55$ & Ile aux Oies & 2 & $\begin{array}{l}\text { M. Moyen } \\
\text { Mme Moyen }\end{array}$ & MI, II: 86. \\
\hline $29-05-55$ & Québec & 1 & Pierre Juneau & Registre de Québec \\
\hline $29-05-55$ & Sillery & 1 & Jean Liégeois & $J J: 196$. \\
\hline $31-07-60$ & Beauport & 1 & Pierre Brincosté 3 & Registre de Québec \\
\hline $6-06-61$ & Tadoussac & 4 & Thomas Michel & JJ: 296. \\
\hline $18-06-61$ & Beaupré $^{4}$ & 5 & & JJ: 298-299. \\
\hline $18-06-61$ & Ile d'Orléans & 5 & & JJ: 298-299. \\
\hline $22-06-61$ & Ile d'Orléans & 7 & $\begin{array}{l}\text { Jean de Lauson } \\
\text { Nicolas Couillard }\end{array}$ & $\begin{array}{l}R J, 1661: 4-5 \\
J J: 298-299 .\end{array}$ \\
\hline & & & $\begin{array}{l}\text { Ignace Sevestre } \\
\text { Elie Jacques } \\
\text { Jacques Perroche } \\
\text { François } \\
\text { Toussaint }\end{array}$ & \\
\hline $11-09-62$ & Ile d'Orléans & 2 & $\begin{array}{l}\text { Jean Leblanc } \\
\text { Gabriel }\end{array}$ & $J J: 311-312$ \\
\hline $10-62$ & Tadoussac & 2 & Couillard des Chênes & $J J: 313$ \\
\hline $14-05-64$ & Québec & 1 & André $^{5}$ & Registre de Québec \\
\hline
\end{tabular}

B. Région de Trois-Rivières

\begin{tabular}{|c|c|c|c|c|}
\hline $2-06-33$ & sur le St-Laurent & 3 & & $R J, 1633: 28$ \\
\hline $09-47$ & sur le St-Laurent & 1 & & $R J, 1647: 73$. \\
\hline $4-07-48$ & Trois-Rivières & 1 & Delachausse & $R J, 1648: 8$ \\
\hline $20-08-50$ & Trois-Rivières & 1 & Robert Lecoq & $J J: 142$ \\
\hline $25-05-51$ & Trois-Rivières & 1 & Noël Godin & $J J: 154$. \\
\hline $7-08-51$ & Trois-Rivières & 1 & Mathurin & $J J: 158$. \\
\hline $10-05-52$ & St-Maurice & 2 & $\begin{array}{l}\text { Jacques Buteux } \\
\text { Fontarabie }\end{array}$ & $R J, 1652: 1-3$ \\
\hline $18-08-52$ & Trois-Rivières & 2 & $\begin{array}{l}\text { Mathurin Guillet } \\
\text { Labouillonnier }\end{array}$ & $R J, 1652: 34$ \\
\hline $19-08-52$ & Trois-Rivières & 8 & $\begin{array}{l}\text { Guillaume Guillemot } \\
\text { du Plessis-Kerbodot } \\
\text { Jean Veron de } \\
\text { Grandmesnil } \\
\text { Matris Belhomme } \\
\text { Guillaume Isabelle } \\
\text { Deslauriers } \\
\text { Lagrave } \\
\text { Langoulmois } \\
\text { Dupuis }\end{array}$ & $\begin{array}{l}J J: 174-175 . \\
R J, 1652: 35 .\end{array}$ \\
\hline
\end{tabular}




\begin{tabular}{|c|c|c|c|}
\hline Date & Lieu de l'incident & Nombre & Noms connus \\
\hline $26-10-52$ & $\begin{array}{l}\text { Cap de la } \\
\text { Madeleine }\end{array}$ & 2 & $\begin{array}{l}\text { Gaillarbois } \\
\text { Saint-Denis }\end{array}$ \\
\hline $3-05-53$ & Trois-Rivières & 2 & \\
\hline $28-05-53$ & Trois-Rivières & 1 & François Lameslé \\
\hline $2-06-53$ & $\begin{array}{l}\text { Cap de la } \\
\text { Madeleine }\end{array}$ & 1 & Cailleteau \\
\hline $23-11-54$ & Trois-Rivières & 1 & $\begin{array}{l}\text { Jean Lanqueteau } \\
\text { Louis Lebecheur } \\
\text { Mathieu Labat } \\
\text { Pierre Chapiteau }\end{array}$ \\
\hline $09-59$ & Trois-Rivières & 1 & Lepine \\
\hline $04-61$ & St-Maurice & 2 & Godefroy fils \\
\hline $8-06-61$ & $\begin{array}{l}\text { Cap de la } \\
\text { Madeleine }\end{array}$ & 3 & \\
\hline
\end{tabular}

$\quad$ Source
$J J: 177$.
$R J: 1653: 6$.
$J J: 181$.
$J J: 182$.
Registre de Trois-
Rivières
Registre de Trois-
Rivières
$J J: 266$.
$R J, 1661: 3-4$
$J J: 297$.

C. Région de Montréal

\begin{tabular}{|c|c|c|c|c|}
\hline $20-08-42$ & Fort Richelieu & 1 & DesLauriers & $R J, 1642: 51$. \\
\hline $9-06-43$ & Montréal & 3 & $\begin{array}{l}\text { Pierre Lafond/ } \\
\text { Laforest } \\
\text { Bertrand Berte } \\
\text { Guillaume Boissier }\end{array}$ & $R J, 1643: 63$ \\
\hline $30-03-44$ & Montréal & 3 & $\begin{array}{l}\text { Guillaume Lebeau } \\
\text { Jean Mattemasse } \\
\text { Pierre Bigot }\end{array}$ & $\begin{array}{l}\text { Registre de Montréal } \\
R J, 1644: 42 .\end{array}$ \\
\hline $7-11-44$ & Fort Richelieu & 1 & & RJ: 1645: 19 \\
\hline $28-07-48$ & Montréal & 1 & Mathurin Bonenfant & Registre de Montréal \\
\hline $6-05-51$ & Montréal & 1 & Jean Boudard & RJ: $1651: 2$ \\
\hline $18-06-51$ & Montréal & 1 & $\begin{array}{l}\text { Léonard Lucos/ } \\
\text { Barbot }\end{array}$ & $J J, 157$ \\
\hline $26-05-52$ & Montréal & 1 & Antoine Roos & Registre de Montréal \\
\hline $16-09-52$ & Montréal & 1 & André David & Registre de Montréal \\
\hline $14-10-52$ & Montréal & 1 & Étienne Thibault & Registre de Montréal \\
\hline $20-07-53$ & Montréal & 1 & Michel Noila & $J J: 185$ \\
\hline $11-10-54$ & Montréal & 1 & Yves Batar & Registre de Montréal \\
\hline $31-05-55$ & Montréal & 1 & Julien Daubigeon & Registre de Montréal \\
\hline $30-08-56$ & vers Lanoraie & 1 & Léonard Gareau & $R J, \quad 1656: 41-43$ \\
\hline $25-10-57$ & Montréal & 3 & $\begin{array}{l}\text { Nicolas Godet } \\
\text { Jean Saint-Père } \\
\text { Jacques Noël }\end{array}$ & $R J, \quad 1658: 3$ \\
\hline $26-10-59$ & Montréal & 1 & Sylvestre Vacher & Registre de Montréal \\
\hline $19-04-60$ & Montréal & 3 & $\begin{array}{l}\text { Nicolas Duval } \\
\text { Mathurin Soulard } \\
\text { Blaise Juillet }\end{array}$ & Registre de Montréal \\
\hline $05-60$ & Long-Sault & 16 & $\begin{array}{l}\text { Adam Dollard } \\
\text { des Ormeaux } \\
\text { François Crusson } \\
\text { Simon Grenet } \\
\text { Jean Lecompte }\end{array}$ & $\begin{array}{l}\text { Registre de Montréal } \\
\text { MI, II: 154-162. }\end{array}$ \\
\hline
\end{tabular}




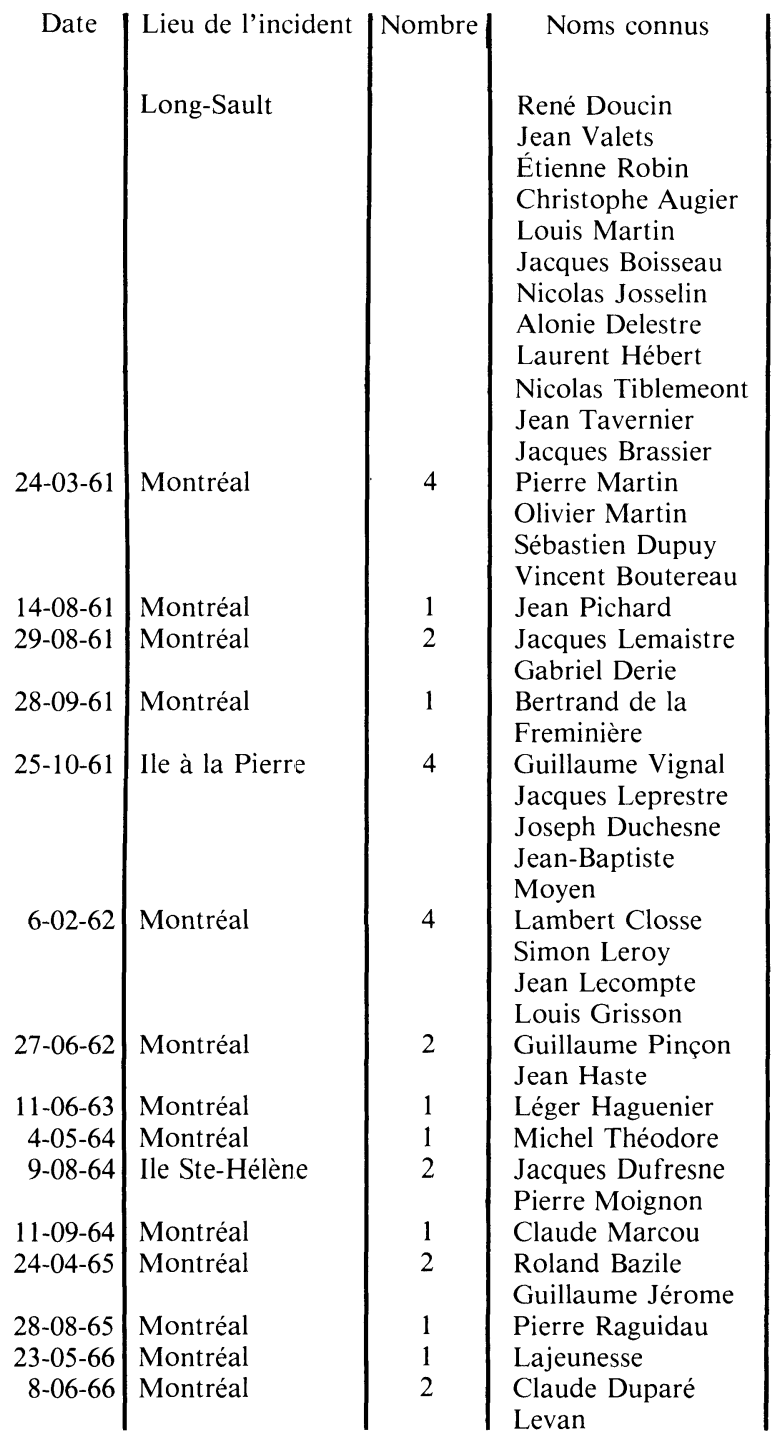

Source

Registre de Montréal

Registre de Montréal $R J$, 1661: 5-6.

Registre de Montréal

$R J, 1665: 20-22$

$R J, 1662: 4-5$.

Registre de Montréal

Registre de Montréal Registre de Montréal Registre de Montréal

Registre de Montréal Registre de Montréal

Registre de Montréal Registre de Montréal Registre de Montréal

D. Ailleurs en Nouvelle-France

\begin{tabular}{r|l|}
$08-27$ & $\begin{array}{l}\text { Chez les Agniers } \\
18-10-46\end{array}$ \\
Chez les Agniers \\
$4-07-48$ & $\begin{array}{l}\text { St-Joseph } \\
\text { des Hurons }\end{array}$ \\
$16-03-49$ & $\begin{array}{l}\text { St-Ignace } \\
\text { des Hurons } \\
7-12-49\end{array}$ \\
$\begin{array}{l}\text { St-Jean-en } \\
\text { Huronie } \\
\text { près de St-Jean }\end{array}$
\end{tabular}

\begin{tabular}{|c|c|}
\hline & Pierre Magnan \\
\hline & $\begin{array}{l}\text { Isaac Jogues } \\
\text { Jean Lalande }\end{array}$ \\
\hline & Antoine Daniel \\
\hline & $\begin{array}{l}\text { Jean de Brébeuf } \\
\text { Gabriel Lalemant }\end{array}$ \\
\hline & Charles Garnier \\
\hline & Noël Chabanel \\
\hline
\end{tabular}

Champlain, III:142-143

$R J$, 1647: 1-3.

$R J$, 1649: 3-5.

$R J$, 1649: 10-17.

$R J, 1650: 8-10$.

$R J, 1650: 16-17$. 
ANNEXE II

Captifs français, 1608-1666

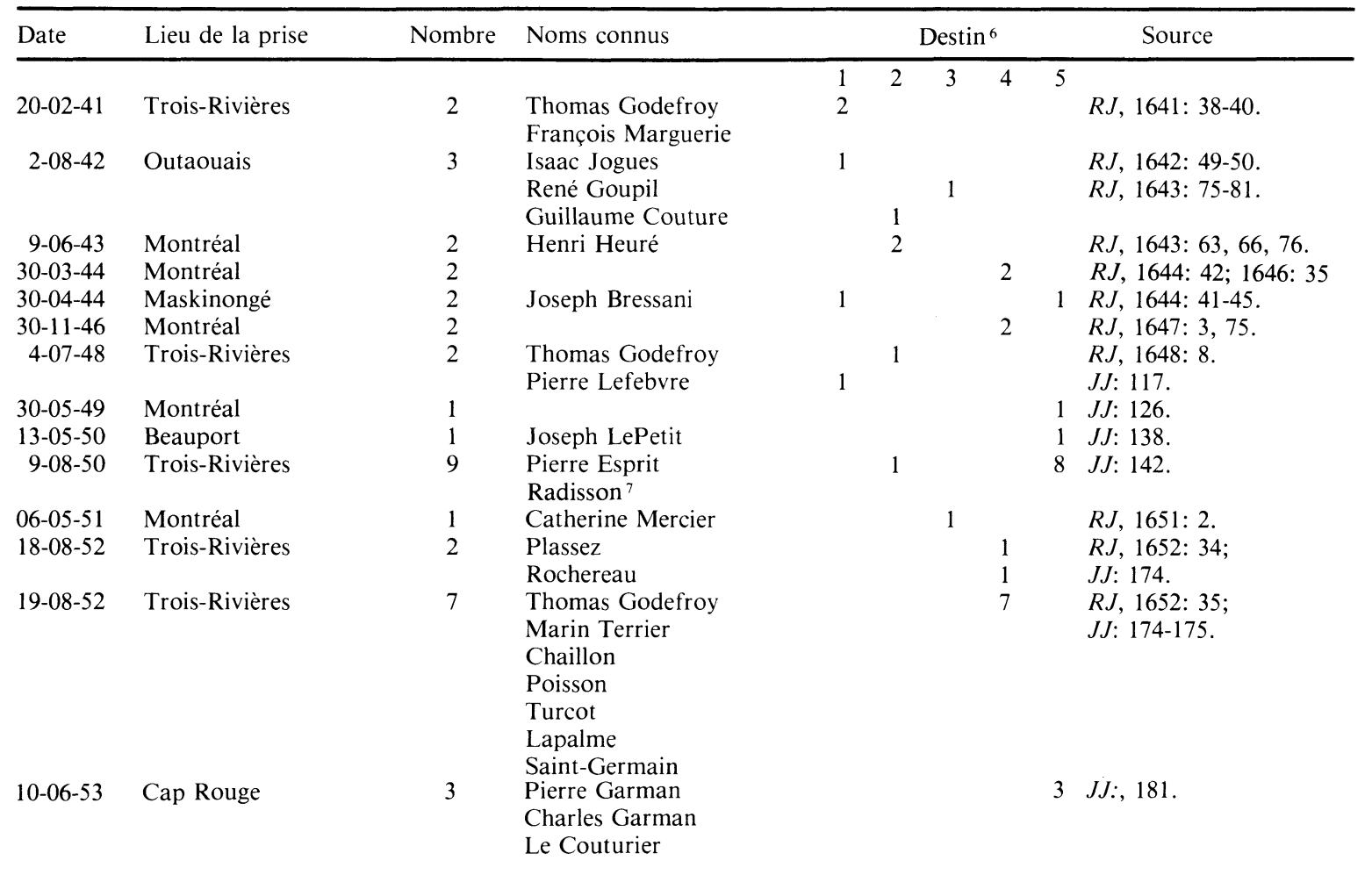




\begin{tabular}{|c|c|c|c|c|c|c|c|c|c|}
\hline Date & Lieu de la prise & Nombre & Noms connus & \multicolumn{5}{|c|}{ Destin } & Source \\
\hline & & & & 1 & 2 & 3 & 4 & 5 & \\
\hline $20-08-53$ & Sillery & 2 & $\begin{array}{l}\text { Joseph Poncet } \\
\text { Mathurin Franchetot }\end{array}$ & 1 & & 1 & & & $R J, 1653: 9-17$. \\
\hline $04-54$ & Montréal & 1 & & 1 & & & & & $R J, 1654: 7-8$ \\
\hline $10-54$ & Montréal & 1 & & 1 & & & & & Ducreux, II: 696 . \\
\hline $27-05-55$ & Île aux Oies & 4 & $\begin{array}{l}2 \text { enfants Moyen } \\
2 \text { enfants Macart }\end{array}$ & 4 & & & & & Ducreux, II: 695-696. \\
\hline $7-05-55$ & Trois-Rivières & 1 & & 1 & & & & & Ducreux, II: 696 . \\
\hline $13-06-58$ & Trois-Rivières & 3 & $\begin{array}{l}\text { Adrien Joliet } \\
\text { Christophe }\end{array}$ & $\begin{array}{l}1 \\
1\end{array}$ & & & & & $\begin{array}{l}R J, 1658: 17 ; \\
J J: 241 .\end{array}$ \\
\hline & & & Fouquet & & & & 1 & & \\
\hline $22-08-58$ & Cap Rouge & 2 & Jean Hayot & & 2 & & & & $R J, 1658: 18$. \\
\hline $5-11-58$ & Trois-Rivières & 4 & & 4 & & & & & $J J: 245-246$ \\
\hline $6-11-58$ & Trois-Rivières & 4 & & 4 & & & & & $J J: 245-246$. \\
\hline $27-06-59$ & Trois-Rivières & 3 & Antoine Des Rosiers & & 2 & 1 & & & $\begin{array}{l}R J, 1659: 2 ; \\
J J: 259,263 .\end{array}$ \\
\hline $25-08-59$ & Trois-Rivières & 8 & & & & & 8 & & $R J, 1659: 2$ \\
\hline $12-09-59$ & Cap Rouge & 1 & Guillaume Routier & & & & 1 & & $J J: 265$. \\
\hline $05-60$ & Long-Sault & 1 & Robert Jurie & & 1 & & & & Registre de Montréal \\
\hline $5-06-60$ & Petit Cap & 5 & $\begin{array}{l}\text { Marie Caron } \\
\text { Louise Picard } \\
\text { Nicolas Picard } \\
\text { Vincent Picart } \\
\text { enfant Picard }\end{array}$ & & $\begin{array}{l}1 \\
1 \\
1 \\
1\end{array}$ & 1 & & & $R J, 1660: 32-33$ \\
\hline $14-08-60$ & Trois-Rivières & 2 & & & & & & 2 & $J J ; 286$. \\
\hline 24-03-61 & Montréal $^{8}$ & 15 & & 25 & & & & 10 & $R J, 1661: 3,7,24-25$, \\
\hline 04-61 & Trois-Rivières & 14 & & & & & & & $33-37 ; R J, 1662: 12 ;$ \\
\hline $18-06-61$ & Beaupré & 3 & & & & & & & $J J ; 295,298,300,320$. \\
\hline $18-06-61$ & Île d'Orléans & 2 & & & & & & & \\
\hline $22-06-61$ & Île d'Orléans & 1 & & & & & & & $R J, 1661: 4-5$. \\
\hline
\end{tabular}




\begin{tabular}{|c|c|c|c|c|c|c|c|c|c|c|}
\hline Date & Lieu de la prise & Nombre & Noms connus & & & estir & & & Source & \\
\hline & & & & 1 & 2 & 3 & 4 & 5 & & \\
\hline \multirow[t]{5}{*}{$24-03-61$} & Montréal & 5 & Pierre Cauvin & & & 1 & & & Registre de Montréal & - \\
\hline & & & Jean Millet & & & 1 & & & Registre de Montréal & 5 \\
\hline & & & Pierre Pitre & & 1 & & & & Registre de Montréal & $\Omega$ \\
\hline & & & Michel Messier & 1 & & & & & Registre de Montréal & 5 \\
\hline & & & Pierre Goguet & 1 & & & & & Registre de Montréal & $\pi$ \\
\hline $3-07-61$ & Trois-Rivières & 1 & François Hertel & & 1 & & & & $R J, 1661: 34-36$ & $\tilde{m}$ \\
\hline $1-08-61$ & Trois-Rivières & 1 & & & 1 & & & & $R J, 1661: 34$ & $\overline{\widetilde{Z}}$ \\
\hline $29-08-61$ & Montréal & 1 & & 1 & & & & & $R J, 1661: 5-6$ & ర \\
\hline \multirow[t]{3}{*}{$25-10-61$} & Montréal & 3 & Claude de Brigeart & & & 1 & & & $R J, 1665: 20-22$ & $\varsigma_{0}$ \\
\hline & & & René Cuillerier & & 1 & & & & & $\sqrt{n}$ \\
\hline & & & Dufresne & 1 & & & & & & \\
\hline \multirow[t]{4}{*}{61} & Trois-Rivières & 4 & Liberté & & & 1 & & & $R J, 1662: 14$ & $\stackrel{\pi}{\rightarrow}$ \\
\hline & & & Antoine de la Meslé & & & 1 & & & $R J, 1661: 35$ & 5 \\
\hline & & & Hébert & & & 1 & & & $R J, 1661: 35$ & 3 \\
\hline & & & Louis Guimont & & & 1 & & & $R J, 1661: 35$ & 0 \\
\hline $06-61$ & Trois-Rivières & 1 & Pierre Rencontre & & & 1 & & & $R J, 1661: 35$ & $\underset{-1}{\pi}$ \\
\hline 62 & Montréal & 1 & Simon Despres dit Berry & & & 1 & & & Registre de Montréal & 2 \\
\hline $12-05-63$ & Montréal & 2 & & & 1 & & & 1 & $R J, 1663: 3-15$ & $\exists$ \\
\hline $10-63$ & Îles du Richelieu & 2 & & 2 & & & & & $R J, 1664: 29-35$ & $\pi$ \\
\hline 64 & Trois-Rivières & 1 & & & & & & 1 & $R J, 1664: 29$ & Z \\
\hline 64 & Île d'Orléans & 1 & & & & & & 1 & $R J, 1664: 29$ & Z \\
\hline $24-04-65$ & Montréal & 2 & $\begin{array}{l}\text { Jacques Petit } \\
\text { Montor }\end{array}$ & & 1 & & & 1 & $\begin{array}{l}\text { Registre de Montréal; } \\
J J: 331 .\end{array}$ & $\stackrel{0}{\complement}$ \\
\hline $09-65$ & Montréal & 1 & LeMoyne & 1 & & & & & $R J, 1666: 3 ; J J: 339$. & 5 \\
\hline 65 & Montréal & 1 & Michel Guibert & & & 1 & & & Registre de Montréal & $\prod$ \\
\hline
\end{tabular}


1 Les sources sont:

$R J$ - Relations des Jésuites (Montréal, Éditions du Jour, 1972).

$J J$ - Charles-Henri Laverdière et Henri-Raymond Casgrain, éds., Journal des Jésuites (Montréal, Editions François Xavier, 1973).

MI - Marie de l'Incarnation, Lettres (abbé Richaudeau éd.) (Paris, Librairie Internationale - Catholique, 1876).

Champlain - Samuel de Champlain, Oeuvres (Montréal, Éditions du Jour, 1973).

Ducreux - François Ducreux, The History of Canada or New France (Percy J. Robinson and James B. Conacher eds.) (Toronto, The Champlain Society, 1951-52).

Il arrive souvent que deux sources mentionnent le même événement. Dans ce cas celle qui donne la meilleure description ou celle qui comporte la meilleure garantie d'exactitude est indiquée.

2 Les victimes des années 1654 et 1655 sont difficiles à identifier à cause des lacunes dans le Journal et les Relations. Ducreux et Marie de l'Incarnation mentionnent le couple Moyen. Il y a un feuillet spécial pour raconter le cas Liégeois dans le Journal. Dans le cas des deux frères Jésuites impliqués dans des incidents, Marie de l'Incarnation est dans l'erreur. Elle indique qu'un frère "qui faisait chemin» fut massacré (II: 86). Il s'agit sans doute du frère Louis Le Boesme blessé aux environs de Sainte-Croix en route pour Trois-Rivières $(J J: 196)$. Mais alors le frère Liégeois n'est pas mentionné. Elle parle de quatre serviteurs de M. Denis qui furent massacrés, mais il n'y a aucune indication chronologique précise. Il est vraisemblable que Boesse et Morin étaient ces serviteurs et que deux autres furent blessés. Les Iroquois ne les avaient pas achevés sur place puisque Morin ne mourut que deux semaines après l'incident.

3 Le registre indique que Pierre Brincosté fut tué par les Iroquois. Cependant c'est un cas douteux car toutes les autres sources insistent sur le fait que l'été ait été paisible après le combat du Long-Sault. Ni le Journal, ni Marie de l'Incarnation ne font mention de ce cas et au contraire l'Ursuline affirme qu'un accident détourna l'armée qui devait venir (II: 189).

4 La répartition des victimes tuées ou capturées sur la côte de Beaupré et l'île d'Orléans est arbitraire - les détails concrets permettant plus de précision manquent. Huit personnes sont impliquées à Beaupré et sept sur l'île d'Orléans le 18 juin 1661 (JJ: 298). Une autre fut prise le 22 juin si la Relation qui affirme que Jean de Lauson était «huitiéme» est exacte $(R J, 1661: 4)$. Le 25 juin on vit les Iroquois au Cap à l'Arbre avec six captifs (JJ: 299). Ainsi dix personnes sur les seize seraient mortes.

5 Seul le registre mentionne cet André qui est peut-être Amérindien.

6 Destin des captifs: 1 - libéré

$$
\begin{aligned}
& 2 \text { - s'évade } \\
& 3 \text { - tué } \\
& 4 \text { - présumé tué } \\
& 5 \text { - inconnu }
\end{aligned}
$$

7 Pierre-Esprit Radisson fut pris à Trois-Rivières entre 1650 et 1652 . Grace Lee Nute indique que sa prise date possiblement de 1651 (Dictionnaire biographique du Canada (Québec, Les Presses de l'Université Laval, 1969), II: 558), mais nous croyons 1650 plus probable puisqu'aucun captif n'est mentionné dans la région trifluvienne en 1651 .

${ }_{8}$ Puisque nous manquons de détails sur les noms des captifs, il est très difficile de reconstruire exactement les faits. Parmi ceux qui n'ont pas pu être identifiés, il est certain que quatre furent libérés le 29 juin $1661(\mathrm{JJ}: 300)$ neuf furent libérés en octobre lors de l'ambassade de Garakontié ( $R J, 1661: 33-37)$; neuf autres revinrent avec le père Le Moine en août $1662(R J, 1662: 12)$; et sept autres arrivèrent dans une barque anglaise le 30 juillet 1663 (JJ: 320). Enfin un homme s'est sauvé dès 1661 (RJ, 1661: 24-25). Parmi ces gens figurent probablement René Cuillerier, Dufresne, Michel Messier et Pierre Goguet. 